\title{
Quantum phases of a chain of strongly interacting anyons
}

\author{
Peter E. Finch, Holger Frahm, Marius Lewerenz, Ashley Milsted, and Tobias J. Osborne \\ Institut für Theoretische Physik, Leibniz Universität Hannover, Appelstraße 2, 30167 Hannover, Germany
}

(Received 14 April 2014; revised manuscript received 4 August 2014; published 19 August 2014)

\begin{abstract}
Quantum gates for the manipulation of topological qubits rely on interactions between non-Abelian anyonic quasiparticles. We study the collective behavior of systems of anyons arising from such interactions. In particular, we study the effect of favoring different fusion channels of the screened Majorana spins appearing in the recently proposed topological Kondo effect. Based on the numerical solution of a chain of $S O(5)_{2}$ anyons we identify two critical phases whose low-energy behavior is characterized by conformal field theories with central charges $c=1$ and $c=8 / 7$, respectively. Our results are complemented by exact results for special values of the coupling constants which provide additional information about the corresponding phase transitions.
\end{abstract}

DOI: 10.1103/PhysRevB.90.081111

PACS number(s): 05.30.Pr, 03.65.Vf, 05.70.Jk

Introduction. Low-dimensional quantum systems hold an irresistible and enduring fascination because they can support topological states of matter with exotic quasiparticles, anyons, exhibiting unusual braiding statistics [1]. While initially a curiosity, anyons generated considerable excitement when it was realized that the fractional quantum Hall effect [2] - and later nanowires $[3,4]$ and the $p_{x}+i p_{y}$ superconductor [5]support these fractionalized excitations. Further interest was recently sparked by the remarkable proposal that systems of non-Abelian anyons could carry out fault-tolerant quantum computation via braiding [6-12].

An increasing number of scenarios for the realization of localized anyon modes in electronic materials have been proposed [13] leading to the recent experimental observation of signatures of Majorana fermions (or Ising anyons) at the ends of nanowires coupled to a superconductor [3,4]. However, braiding of Ising anyons alone does not yield a set of gates sufficient for universal quantum computation: topological qubits are found by additionally coupling $M$ of these quasiparticles to form an $S O(M)$ Majorana spin.

When connected to electronic leads via tunnel junctions Majorana topological spins exhibit the recently proposed "topological" Kondo effect displaying strong non-Fermiliquid correlations [14]. Exploiting a combination of both perturbative renormalization group analysis and conformal field theory methods the corresponding Kondo fixed point has been identified with an $S O(M)_{2}$ Wess-Zumino-Novikov-Witten boundary conformal field theory $[14,15]$. A proposed scheme to probe and manipulate these Kondo-screened Majorana spins $[15,16]$ constitutes a first step towards a realization of quantum gates. Indeed it was shown that $\mathrm{SO}(3)_{2}$ anyons [and likely $S O(p)_{2}$ for prime $p \geqslant 5$ ] are able to support universal quantum computation [17]. Thus the study of models for $S O(M)_{2}$ anyons has become an intriguing and relevant possibility and introducing couplings between such objects is now a natural next step.

Unfortunately systems of anyons are extremely difficult to study: One must keep track of their entire space-time history in order to discuss their dynamics. While the noninteracting case is now becoming well understood (see, e.g., [10]) the classification of phases for systems of interacting anyons has progressed much slower. An additional complication is that the description of the dynamics of a highly entangled $S O(M)$ Majorana spin in the topological Kondo model, and the collective behaviour of many such subsystems, involves dealing with strong correlations. Indeed, only recently have one-dimensional interacting systems, where the anyon model may be seen as a deformation of $S U(2)$, been studied in earnest. Using exact numerical diagonalization, partly complemented by analytical results known for the related restricted solid on solid models [18,19], it has been possible to identify the possible phases realized in these models [20,21]. These models are not just toys: The study of one-dimensional systems offers nontrivial insight into the more general case as they mediate the boundary between different topological phases [22].

In one dimension we can exploit powerful tensor-network variational methods falling under the umbrella of the density matrix renormalization group $[23,24]$. These methods, with impetus from the study of quantum entanglement, have led to unparalleled insights in recent years overcoming many previously insurmountable roadblocks, including the simulation of dynamics [25,26] and fermions [27-30] without sign problems, the determination of spectral information [31], and higher dimensions by exploiting tensor networks including the projected entangled-pair states [32] and the multiscale entanglement renormalization ansatz (MERA) [33,34]. This progress has been recently extended to the anyonic setting with the development of anyonic matrix product states (MPS) and MERA routines to obtain ground-state properties [35,36] and to simulate the spectrum and dynamics of interacting anyons [37-40], providing an attractive complement to other methods such as exact diagonalization [22,41-43] and Monte Carlo [44].

The purpose of this Rapid Communication is to exploit both analytical and numerical tools to carry out a comprehensive study of a nontrivial system of anyons relevant to the topological Kondo effect. Specifically, as an effective model, we consider the fundamental quasiparticles in an $S O(5)_{2}$ Chern-Simons theory [45]. Using two complementary techniques, one based on the Bethe ansatz and the other on cutting-edge tangent-plane tensor network methods, we investigate the ground-state and low-lying excitations of a one-dimensional condensate of these non-Abelian anyons and characterize and classify their critical phases and integrable points.

The fusion rules for $S O(5)_{2}$, the truncation of the category of irreducible representations of the quantum group $U_{q}[s o(5)]$, 
TABLE I. Fusion rules for the $S O(5)_{2}$ anyons.

\begin{tabular}{lccccc}
\hline \hline$\otimes$ & $\psi_{1}$ & $\psi_{2}$ & $\psi_{3}$ & $\psi_{4}$ & $\psi_{5}$ \\
\hline$\psi_{1}$ & $\psi_{1}$ & $\psi_{2}$ & $\psi_{3}$ & $\psi_{5}$ & $\psi_{5}$ \\
$\psi_{2}$ & $\psi_{2}$ & $\psi_{1} \oplus \psi_{5} \oplus \psi_{6}$ & $\psi_{3} \oplus \psi_{4}$ & $\psi_{3} \oplus \psi_{4}$ & $\psi_{2} \oplus \psi_{5}$ \\
$\psi_{3}$ & $\psi_{3}$ & $\psi_{3} \oplus \psi_{4}$ & $\psi_{1} \oplus \psi_{2} \oplus \psi_{5}$ & $\psi_{2} \oplus \psi_{5} \oplus \psi_{6}$ & $\psi_{3} \oplus \psi_{4}$ \\
$\psi_{4}$ & $\psi_{4}$ & $\psi_{3} \oplus \psi_{4}$ & $\psi_{2} \oplus \psi_{5} \oplus \psi_{6}$ & $\psi_{1} \oplus \psi_{2} \oplus \psi_{5}$ & $\psi_{3} \oplus \psi_{4}$ \\
$\psi_{5}$ & $\psi_{5}$ & $\psi_{2} \oplus \psi_{5}$ & $\psi_{3} \oplus \psi_{4}$ & $\psi_{3} \oplus \psi_{4}$ & $\psi_{1} \oplus \psi_{2} \oplus \psi_{6}$ \\
$\psi_{6}$ & $\psi_{6}$ & $\psi_{2}$ & $\psi_{4}$ & $\psi_{3}$ & $\psi_{5}$ \\
\hline \hline
\end{tabular}

with $q=e^{2 i \pi / 5}$ [46], are given in Table I. They are diagonalized by the modular $S$ matrix

$$
S=\frac{1}{2 \sqrt{5}}\left(\begin{array}{cccccc}
1 & 2 & \sqrt{5} & \sqrt{5} & 2 & 1 \\
2 & -2 \phi & 0 & 0 & 2 \phi^{-1} & 2 \\
\sqrt{5} & 0 & -\sqrt{5} & \sqrt{5} & 0 & -\sqrt{5} \\
\sqrt{5} & 0 & \sqrt{5} & -\sqrt{5} & 0 & -\sqrt{5} \\
2 & 2 \phi^{-1} & 0 & 0 & -2 \phi & 2 \\
1 & 2 & -\sqrt{5} & -\sqrt{5} & 2 & 1
\end{array}\right),
$$

where $\phi=\frac{1+\sqrt{5}}{2}$. Specifically, we consider fusion paths of length $\mathcal{L}$ for $\psi_{3}$ anyons, represented diagrammatically as

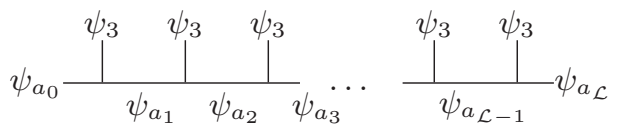

where fusing occurs from top left to bottom right. Below these paths are identified with basis vectors of an anyonic Hilbert space, i.e., $\left|a_{0} a_{1} \ldots a_{\mathcal{L}}\right\rangle$, where neighboring labels must be related through fusion with $\psi_{3}$. This is equivalent to labels $a_{i}$ and $a_{i+1}$ being adjacent on following graph:

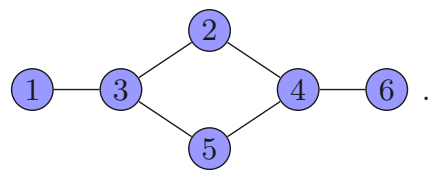

Ordering of the fusion can be changed by means of $F$ moves (i.e., generalized 6- $j$ symbols),

$\left.\psi_{a_{i-1}}\right|_{\psi_{a_{i}}} ^{\psi_{3}} \psi_{a_{i+1}} \psi_{3}=\sum_{a_{i}^{\prime}}\left(F_{a_{i+1}}^{a_{i-1} 33}\right)_{a_{i}^{\prime}}^{a_{i}} \psi_{a_{i-1}} \underbrace{\psi_{3}}_{\psi_{i}^{\prime}} \psi_{a_{i+1} .}$

For the $S O(5)_{2}$ fusion rules there exist four known sets of inequivalent unitary $F$ moves, each of which corresponds to a different $S$ matrix [46]. Hence our choice (1) of the $S$ matrix determines the $F$ moves which can be used to construct two-site projection operators,

$$
\begin{aligned}
p_{i}^{(b)}= & \sum_{a_{i-1}, a_{i}, a_{i}^{\prime}, a_{i+1}}\left[\left(F_{a_{i+1}}^{a_{i-1} j j}\right)_{b}^{a_{i}^{\prime}}\right]^{*}\left(F_{a_{i+1}}^{a_{i-1} j j}\right)_{b}^{a_{i}} \\
& \times\left|\ldots a_{i-1} a_{i}^{\prime} a_{i+1} \ldots\right\rangle\left\langle\ldots a_{i-1} a_{i} a_{i+1} \ldots\right| .
\end{aligned}
$$

We can couple pairs of $\mathrm{SO}(5)_{2}$ anyons via these projection operators leading to a chain of $S O(5)_{2}$ anyons with nearestneighbor interactions subject to periodic boundary conditions $a_{0} \equiv a_{\mathcal{L}}$

$$
\mathcal{H}_{\theta}=\sum_{i=1}^{\mathcal{L}}\left[\cos \left(\frac{\pi}{4}+\theta\right) p_{i}^{(2)}+\sin \left(\frac{\pi}{4}+\theta\right) p_{i}^{(5)}\right] .
$$

From the fusion rules we see that there exists an automorphism exchanging $\psi_{2}$ and $\psi_{5}$ which allows one to construct a nonlocal unitary transformation mapping $\mathcal{H}_{\theta} \leftrightarrow \mathcal{H}_{-\theta}$.

An important property of anyonic models of this type is the presence of topological charges that commute with each other and the global Hamiltonian:

$$
\left\langle a_{1}^{\prime} \ldots a_{\mathcal{L}}^{\prime}\left|Y_{\ell}\right| a_{1} \ldots a_{\mathcal{L}}\right\rangle=\prod_{i=1}^{\mathcal{L}}\left(F_{a_{i+1}}^{\ell a_{i}^{\prime} 3}\right)_{a_{i+1}^{\prime}}^{a_{i}} .
$$

The action of these operators corresponds to the insertion of an auxiliary anyon $\psi_{\ell}$, which is then moved around the ring by application of $F$ moves, and finally removed again [9,21]. The eigenvalues of $Y_{\ell}$ are given in terms of the elements of the $S$ matrix (1) as $\frac{S_{j \ell}}{S_{1 \ell}}[9]$.

To study the model (3) we have employed a combination of numerical and analytical techniques. We exploit the symmetry under exchange of $\psi_{2}$ and $\psi_{5}$ mentioned above as an additional check for our numerical results.

Numerical analysis. We simulated the $\psi_{3}$ anyon chain numerically using the EVOMPS software package [47] via the time-dependent variational principle (TDVP) in imaginary time to calculate an approximate matrix product state representation of the ground state $[48,49]$. The TDVP approximately solves the Schrödinger equation for an infinitesimal time step, providing flow equations for the MPS variational parameters [26]. In imaginary time the convergence of these flow equations can be enhanced via the nonlinear conjugate gradient method [50].

For this work we used uniform MPS to represent translation-invariant states of the chain in the thermodynamic limit. (Note that here a "site" in the chain corresponds to a label in the fusion path, not a site where a $\psi_{3}$ anyon sits.) Since the system is invariant under translations of two sites, we blocked adjacent labels into a virtual site. We also exploit a symmetry which allows us to only consider states with labels 1,2,5,6 on odd sites and labels 3,4 on even sites. This simplification results in a local dimension of 6 for the virtual sites.

A state constructed in this way is still not generally a state of the anyon chain, because it is only guaranteed to be compatible with the fusion rules within a single virtual site: We added penalty terms to the Hamiltonian to suppress states not corresponding to a valid fusion path. 


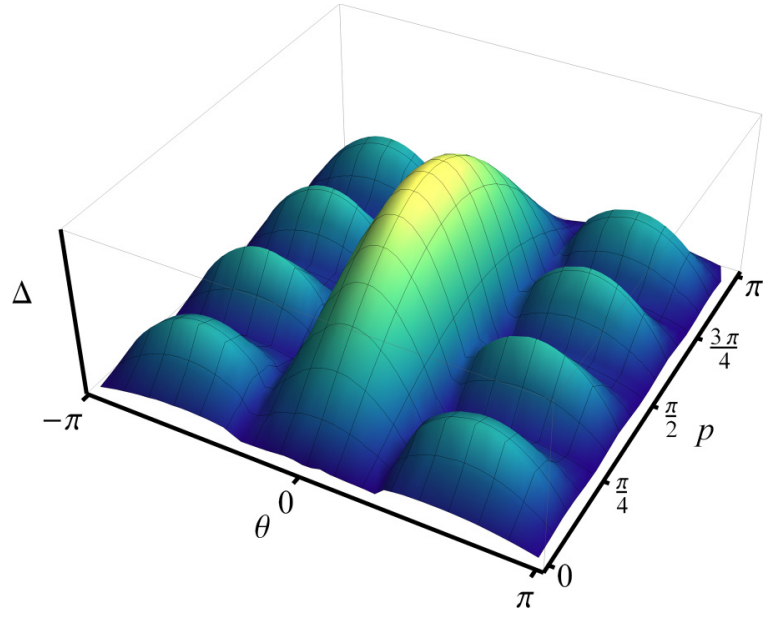

FIG. 1. (Color online) Energies of excited states for the chain in the thermodynamic limit: Between momentum zero and $\pi$, two distinct regions can be observed. The symmetry around zero is apparent, as well as a periodicity for the high $|\theta|$ regions, which indicates a breakdown of translation invariance.

Tangent-plane methods allow for the easy calculation of the energies of low-lying excited states via an MPS ansatz for excited states [31]. The resulting lowest energies are visualized in Fig. 1.

Conformal invariance allows one to characterize the collective behavior of the anyons for parameters where the model supports massless excitations in terms of the central charge $c$ of the underlying Virasoro algebra. From our numerical data it may be extracted from the finite-entanglement scaling behavior (which fulfills a role comparable to that of finite-size scaling) of the entanglement entropy $S$ as a function of the correlation length $\xi$. Although both quantities diverge for the exact ground state of a critical system, they remain finite for a uniform MPS approximation at finite bond dimension, which enforces exponential decay of correlations. Their scaling has been shown to be determined by the central charge $[51,52]$ and is described by [53]

$$
S(\xi) \propto \frac{c}{6} \log \xi
$$

where $S$ and $\xi$ are functions of $D$ that are easily obtained from the MPS representation. An exemplary result for this scaling behavior is shown in Fig. 2(a). We use this relation to estimate central charges for the model [Fig. 2(b)].

Using the central charge and dispersion relations we obtained a phase portrait (Fig. 3), from which we see that there exist two large regions, $\frac{\pi}{3} \lesssim|\theta|<\pi$, with central charge $c=$ 1 and two small intervals near $\theta \approx \pm 0.033 \pi$ where the central charge of the model is found to be $c=8 / 7$. These results agree with the analytic results at two of the integrable points discussed below. Beyond these regions we got spurious results, possibly due to local energy minima, or due to especially strong corrections to the asymptotic relation (5), and it was not possible to determine a consistent central charge. Between the $c=1$ sectors at $\theta=\pi$ the spectrum shows level crossings with large degeneracies, indicating a first order transition. This is consistent with a fixed point of the $\psi_{2} \leftrightarrow \psi_{5}$ automorphism.

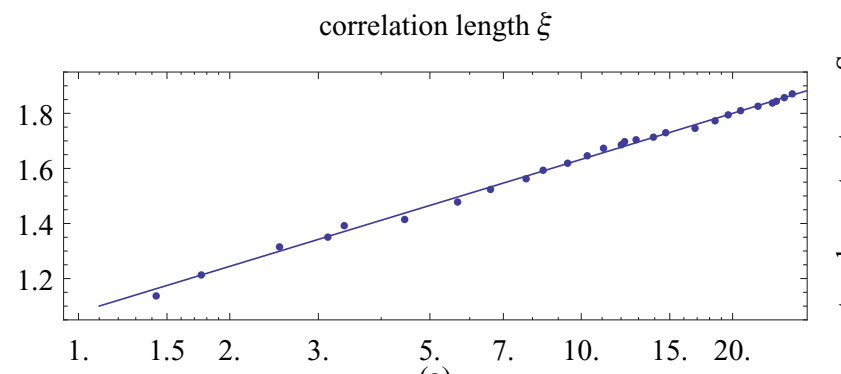

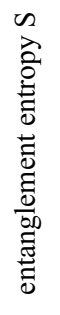

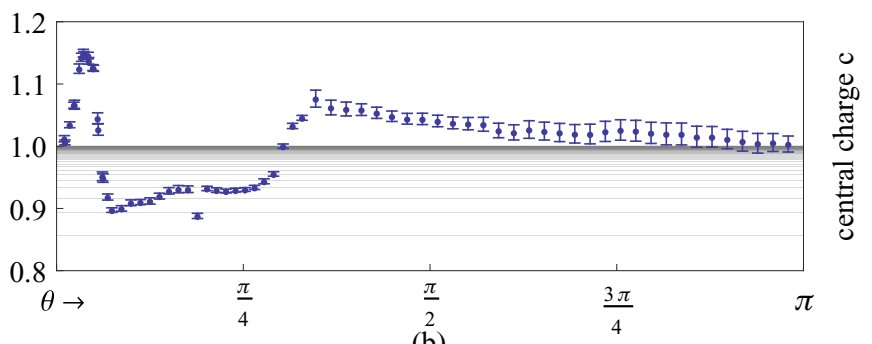

(b)

FIG. 2. (Color online) (a) shows an exemplary entropy curve for $\theta=3.08$ which lies within the $c=1$ region. It also includes a fit with $c=1.0035 \pm 0.012$. (b) shows fitted values for the central charge. The error bars included in the figure represent only the standard deviation based on the fit and do not include errors in the state, for example due to reaching a local minimum within the variational approach. The horizontal lines mark allowed central charges $c<1$ for the unitary minimal models.

Finally, near $\theta=0$, our analytical approach predicts a small gapped region (see below).

Integrable points. The numerical investigation can be complemented by analytical results for special values of the coupling parameter for which the model becomes integrable. The identification of integrable points and associated $R$ matrices solving the Yang-Baxter equation (YBE) is achieved though the realization that the projection operators (2) form

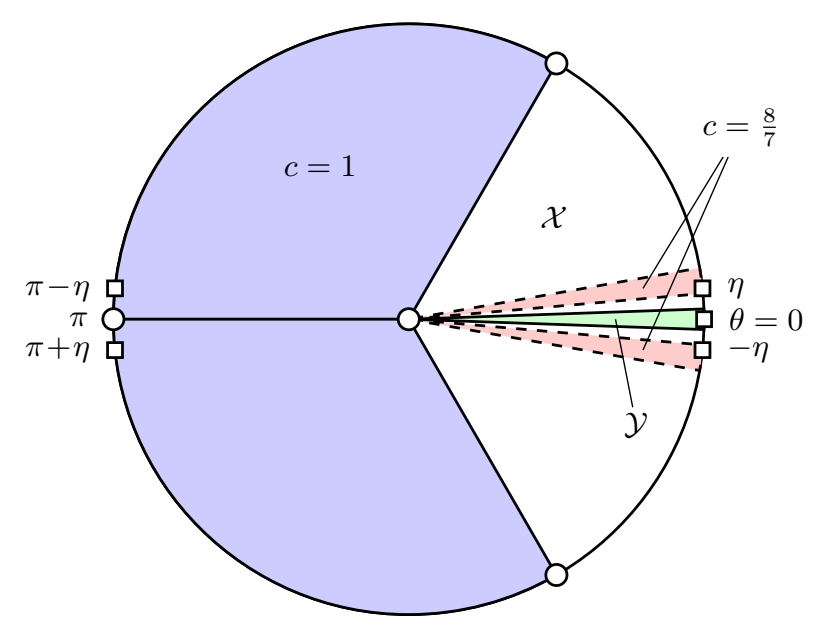

FIG. 3. (Color online) Phase diagram for the $\mathrm{SO}(5)_{2} \psi_{3}$ chain derived from numerical (MPS) results and including integrable points at $|\theta|=0, \eta, \pi-\eta, \pi$. We do not have consistent results for region $\mathcal{X}$, where the central charge appears to be less than 1 . Bethe ansatz results indicate that region $\mathcal{Y}$ is gapped. 
a representation of the Birman-Murakami-Wenzl (BMW) algebra [54]. In particular, choosing $\theta=0, \pi$, the Hamiltonian can be written as

$$
\mathcal{H}_{0, \pi}=\frac{\mp 1}{\sqrt{10}} \sum_{i=1}^{\mathcal{L}} u_{i}+\text { const }
$$

where the $u_{i}=\sqrt{5} p_{i}^{(1)}$ generate a representation of a subalgebra of the BMW algebra isomorphic to the periodic Temperley-Lieb algebra [55]. This model can be derived from an $R$ matrix

$$
R(u)=w_{1}(u) p^{(1)}+w_{2}(u) p^{(2)}+w_{5}(u) p^{(5)},
$$

with weights $w_{1}(u)=\sinh (\gamma-u)$ and $w_{2}(u)=\sinh (\gamma+$ $u)=w_{5}(u)$, where $\cosh \gamma=\frac{\sqrt{5}}{2}$. The global Hamiltonian (3) can be obtained as the logarithmic derivative of a commuting transfer matrix built from this $R$ matrix.

As a consequence of the underlying Temperley-Lieb algebra the spectrum of the model at $\theta=0, \pi$ can be related to that of the $X X Z$ spin-1/2 chain with anisotropy $\pm \Delta=\cosh \gamma=$ $\frac{\sqrt{5}}{2}[55,56]$. This implies that the eigenstates of the model can be parametrized by solutions $\left\{u_{j}\right\}_{j=1}^{n}$ to the Bethe equations associated with the $X X Z$ model for suitably twisted boundary conditions

$$
\left[\frac{\sinh \left(u_{j}-\frac{\gamma}{2}\right)}{\sinh \left(u_{j}+\frac{\gamma}{2}\right)}\right]^{\mathcal{L}}=-\zeta^{-2} \prod_{k=1}^{n} \frac{\sinh \left(u_{j}-u_{k}-\gamma\right)}{\sinh \left(u_{j}-u_{k}+\gamma\right)} .
$$

The twist $\zeta$ appearing in the Bethe equations depends on the sector $n$ : for $n=\frac{\mathcal{L}}{2}$ it can take values $\pm i, e^{ \pm 2 i \pi / 3}, e^{ \pm \gamma}$ while $\zeta^{\mathcal{L}-(n / 2)}=1$ otherwise. This is similar to other higher spin Temperley-Lieb chains [57]. The bulk properties of the quantum chain can be obtained from the equivalence to the $X X Z$ chain: $\theta=0$ corresponds to the antiferromagnetic spin chain which has a tiny energy gap of $\Delta=\pi \exp \left(-\frac{\pi^{2}}{2 \gamma}\right) /(\gamma \sqrt{10}) \simeq$ $2.9 \times 10^{-4}$ [58]. This gap is too small to be resolved in our numerical results but based on continuity arguments we expect that there is an extended massive region in the phase diagram of the anyon chain around $\theta=0$. As a consequence of the multiple twists in the anyon chain the thermodynamic ground state of this model is tenfold degenerate.

At $\theta=\pi$ the Temperley-Lieb equivalence is to the massive ferromagnetic $X X Z$ chain. The ground states have energy $E=-\frac{1}{\sqrt{2}} \mathcal{L}$ and all possible momenta. We also find that there is a large range of excitations lying above the gap which are characterized by the parameters $l, k \in \mathbb{Z}$. The energy and momentum of these states are given by

$$
\begin{aligned}
\Delta E & =\frac{1}{\sqrt{2}}-\frac{2}{\sqrt{10}} \cos \left(\frac{2 k \pi}{\mathcal{L}}-\frac{4 l \pi}{\mathcal{L}(\mathcal{L}-2)}\right), \\
P & =\frac{2 \pi}{\mathcal{L}}(k+l) .
\end{aligned}
$$

As observed in the numerical analysis the spectrum of the anyon chain shows degeneracies growing with the system size indicating a first order transition at $\theta=\pi$.

We have identified two additional pairs of coupling constants where the anyon model becomes integrable: for $\theta=$ $\eta, \pi+\eta$ with $\eta=-\frac{\pi}{4}+\operatorname{atan}\left(\frac{1+\sqrt{5}}{4}\right)$ the Hamiltonian (3) can be obtained from an $R$ matrix associated with the full BMW algebra which is given by (6) with weights

$$
\begin{aligned}
& w_{1}(u)=\sinh \left(u+\frac{i \pi}{10}\right) \sinh \left(u+\frac{3 i \pi}{10}\right)=w_{2}(-u), \\
& w_{5}(u)=\sinh \left(u+\frac{9 i \pi}{10}\right) \sinh \left(u+\frac{3 i \pi}{10}\right) .
\end{aligned}
$$

A third solution to the YBE is related to (7) by the transformation mapping $\theta \leftrightarrow-\theta$ mentioned above.

For both of these solutions we can construct a set of six commuting transfer matrices $t^{(\ell)}(u), \ell=1, \ldots, 6$, whose asymptotics as $u \rightarrow \pm \infty$ is given by the topological charges (4) up to a scalar function. This implies that the charges $Y_{\ell}$ are elements of the algebra of commuting integrals of the anyon chain. By diagonalizing the transfer matrix $t^{(3)}(u)$ we find that the spectrum of the anyon chain at these integrable points can be obtained from

$$
\left[i \frac{\sinh \left(u_{j}+\frac{i \pi}{20}\right)}{\sinh \left(u_{j}-\frac{i \pi}{20}\right)}\right]^{\mathcal{L}}=-\zeta \prod_{k=1}^{n} \frac{\sinh \left(u_{j}-u_{k}+\frac{2 i \pi}{5}\right)}{\sinh \left(u_{j}-u_{k}-\frac{2 i \pi}{5}\right)},
$$

where the $\zeta= \pm 1$ is the eigenvalue of $Y_{6}$. Note that these Bethe equations are (up to a twist) those of the $\mathbb{Z}_{5}$ FateevZamolodchikov (FZ) model, sometimes also referred to as the five-state self-dual chiral Potts model $[59,60]$. This can be understood as a consequence that the $R$ matrices with (7) correspond to descendants of the zero-field six-vertex model [61].

Solving the Bethe equations (8) we have analyzed the ground-state and low-lying excitations of the anyon chain in the thermodynamic limit and found that these integrable points are described by effective conformal field theories with central charges $c=\frac{8}{7}$ for $\theta= \pm \eta$ and $c=1$ for $\pi \pm \eta$, just as the ferro- (antiferro-)magnetic $\mathbb{Z}_{5} \mathrm{FZ}$ model [60]. Based on the $S$ matrix (1) and the operator content of the anyon chain as identified from our finite-size analysis of the spectrum we conjecture, however, that the low-energy theories are unitary rational models invariant under extensions of the Virasoro algebra, i.e., the $\mathcal{W} B_{2}\left(\mathcal{W} D_{5}\right)$ algebra for $\theta= \pm \eta(\pi \pm \eta)$. An extended and more formal treatment of the integrable points will be presented in a separate publication [62].

Conclusion. We introduced a lattice model of the anyons expected to be relevant for the low-energy physics of the $M=5$ topological Kondo effect. The degeneracy of the anyon zero modes is lifted by local interactions consistent by the $S O(5)_{2}$ fusion rules. We have been able to characterize the collective states of the resulting strongly interacting anyon model by a combination of numerical simulation and analytic results. We found multiple extended critical regions with central charges $c=1$ and $c=8 / 7$, which were calculated from finite-entanglement scaling. In addition we were able to identify the low-energy effective field theory for special values of the coupling from the Bethe ansatz solution.

Acknowledgments. The authors would like to thank Eddy Ardonne and Michael Flohr for helpful discussions. This work was supported by the Deutsche Forschungsgemeinschaft under grant no. Fr-737/7, by the ERC grants QFTCMPS and SIQS, and by the cluster of excellence EXC 201 Quantum Engineering and Space-Time Research. 
[1] F. Wilczek, Fractional Statistics and AnyonSuperconductivity (World Scientific, Teaneck, NJ, 1990), pp. x+447.

[2] G. Moore and N. Read, Nucl. Phys. B 360, 362 (1991); N. Read and E. Rezayi, Phys. Rev. B 59, 8084 (1999).

[3] T. D. Stanescu, R. M. Lutchyn, and S. Das Sarma, Phys. Rev. B 84, 144522 (2011).

[4] V. Mourik, K. Zuo, S. M. Frolov, S. R. Plissard, E. P. A. M. Bakkers, and L. P. Kouwenhoven, Science 336, 1003 (2012).

[5] N. Read and D. Green, Phys. Rev. B 61, 10267 (2000).

[6] E. Dennis, A. Kitaev, A. Landahl, and J. Preskill, J. Math. Phys. 43, 4452 (2002).

[7] C. Nayak, S. H. Simon, A. Stern, M. Freedman, and S. Das Sarma, Rev. Mod. Phys. 80, 1083 (2008).

[8] A. Y. Kitaev, Ann. Phys. 303, 2 (2003).

[9] A. Kitaev, Ann. Phys. 321, 2 (2006).

[10] Z. Wang, Topological Quantum Computation (American Mathematical Society, Providence, 2008).

[11] J. K. Pachos, Introduction to Topological Quantum Computation (Cambridge University Press, Cambridge, 2012).

[12] R. Barends et al., Nature (London) 508, 500 (2014).

[13] C. W. J. Beenakker, Annu. Rev. Condens. Mattter Phys. 4, 113 (2013).

[14] B. Béri and N. R. Cooper, Phys. Rev. Lett. 109, 156803 (2012).

[15] A. Altland, B. Béri, R. Egger, and A. M. Tsvelik, Phys. Rev. Lett. 113, 076401 (2014).

[16] A. Altland, B. Béri, R. Egger, and A. M. Tsvelik, J. Phys. A 47, 265001 (2014).

[17] S. X. Cui and Z. Wang, arXiv:1405.7778.

[18] G. E. Andrews, R. J. Baxter, and P. J. Forrester, J. Stat. Phys. 35, 193 (1984).

[19] P. Kakashvili and E. Ardonne, Phys. Rev. B 85, 115116 (2012).

[20] A. Feiguin, S. Trebst, A. W. W. Ludwig, M. Troyer, A. Kitaev, Z. Wang, and M. H. Freedman, Phys. Rev. Lett. 98, 160409 (2007).

[21] C. Gils, E. Ardonne, S. Trebst, D. A. Huse, A. W. W. Ludwig, M. Troyer, and Z. Wang, Phys. Rev. B 87, 235120 (2013).

[22] C. Gils, E. Ardonne, S. Trebst, A. W. W. Ludwig, M. Troyer, and Z. Wang, Phys. Rev. Lett. 103, 070401 (2009).

[23] U. Schollwöck, Rev. Mod. Phys. 77, 259 (2005).

[24] U. Schollwöck, Ann. Phys. 326, 96 (2011).

[25] G. Vidal, Phys. Rev. Lett. 93, 040502 (2004).

[26] J. Haegeman, J. I. Cirac, T. J. Osborne, I. Pižorn, H. Verschelde, and F. Verstraete, Phys. Rev. Lett. 107, 070601 (2011).

[27] P. Corboz and G. Vidal, Phys. Rev. B 80, 165129 (2009).

[28] P. Corboz, G. Evenbly, F. Verstraete, and G. Vidal, Phys. Rev. A 81, 010303 (2010).

[29] P. Corboz, R. Orús, B. Bauer, and G. Vidal, Phys. Rev. B 81, 165104 (2010).

[30] C. V. Kraus, N. Schuch, F. Verstraete, and J. I. Cirac, Phys. Rev. A 81, 052338 (2010).
[31] J. Haegeman, B. Pirvu, D. J. Weir, J. I. Cirac, T. J. Osborne, H Verschelde, and F. Verstraete, Phys. Rev. B 85, 100408 (2012).

[32] F. Verstraete and J. I. Cirac, arXiv:cond-mat/0407066.

[33] G. Vidal, Phys. Rev. Lett. 101, 110501 (2008).

[34] G. Vidal, Phys. Rev. Lett. 99, 220405 (2007).

[35] R. König and E. Bilgin, Phys. Rev. B 82, 125118 (2010).

[36] R. N. C. Pfeifer, P. Corboz, O. Buerschaper, M. Aguado, M. Troyer, and G. Vidal, Phys. Rev. B 82115126 (2010).

[37] S. Singh, R. N. C. Pfeifer, G. Vidal, and G. K. Brennen, Phys. Rev. B 89, 075112 (2014).

[38] D. Poilblanc, A. Feiguin, M. Troyer, E. Ardonne, and P. Bonderson, Phys. Rev. B 87, 085106 (2013).

[39] V. Zatloukal, L. Lehman, S. Singh, J. K. Pachos, and G. K. Brennen, arXiv:1207.5000.

[40] M. P. Zaletel, R. S. K. Mong, and F. Pollmann, Phys. Rev. Lett. 110, 236801 (2013).

[41] S. Trebst, E. Ardonne, A. Feiguin, D. A. Huse, A. W. W. Ludwig, and M. Troyer, Phys. Rev. Lett. 101, 050401 (2008).

[42] D. Poilblanc, A. W. W. Ludwig, S. Trebst, and M. Troyer, Phys. Rev. B 83, 134439 (2011).

[43] D. Poilblanc, M. Troyer, E. Ardonne, and P. Bonderson, Phys. Rev. Lett. 108, 207201 (2012).

[44] H. Tran and N. Bonesteel, Comput. Mater. Sci. 49, S395 (2010).

[45] M. B. Hastings, C. Nayak, and Z. Wang, Phys. Rev. B 87, 165421 (2013).

[46] P. H. Bonderson, Ph.D. thesis, California Institute of Technology, 2007.

[47] A. Milsted, EVOMPS source code, 2013, https://github.com/ amilsted/evoMPS.

[48] M. Fannes, B. Nachtergaele, and R. F. Werner, Commun. Math. Phys. 144, 443 (1992).

[49] F. Verstraete, V. Murg, and J. Cirac, Adv. Phys. 57, 143 (2008).

[50] A. Milsted, J. Haegeman, and T. J. Osborne, Phys. Rev. D 88, 085030 (2013).

[51] L. Tagliacozzo, T. R. de Oliveira, S. Iblisdir, and J. I. Latorre, Phys. Rev. B 78, 024410 (2008).

[52] V. Stojevic, J. Haegeman, I. P. McCulloch, L. Tagliacozzo, and F. Verstraete, arXiv:1401.7654.

[53] P. Calabrese and J. Cardy, J. Stat. Mech. (2004) P06002.

[54] J. S. Birman and H. Wenzl, Trans. Amer. Math. Soc. 313, 249 (1989); J. Murakami, Osaka J. Math. 24, 745 (1987).

[55] H. N. V. Temperley and E. H. Lieb, Proc. R. Soc. London, Ser A 332, 251 (1971).

[56] A. L. Owczarek and R. J. Baxter, J. Stat. Phys. 49, 1093 (1987).

[57] B. Aufgebauer and A. Klümper, J. Stat. Mech. (2010) P05018.

[58] J. D. Cloizeaux and M. Gaudin, J. Math. Phys. 7, 1384 (1966).

[59] V. A. Fateev and A. B. Zamolodchikov, Phys. Lett. A 92, 37 (1982).

[60] G. Albertini, Int. J. Mod. Phys. A 9, 4921 (1994).

[61] V. V. Bazhanov and Yu. G. Stroganov, J. Stat. Phys. 59, 799 (1990).

[62] P. E. Finch, M. Flohr, and H. Frahm, arXiv:1408.1282. 